\title{
RM due to magnetic fields in the cosmic web and SKA observations
}

\author{
Takuya Akahori ${ }^{1,2}$ and Dongsu Ryu ${ }^{3}$ \\ ${ }^{1}$ Korea Astronomy and Space Science Institute, Daejeon 305-348, Korea, akahori@kasi.re.kr \\ ${ }^{2}$ School of Physics, The University of Sydney, NSW 2006, Australia \\ ${ }^{3}$ Chungnam National University Daejeon 305-764, Korea, ryu@canopus.cnu.ac.kr
}

\begin{abstract}
We estimated that rotation measure (RM) due to the intergalactic magnetic field (IGMF) in the cosmic web is $\sim 1-10 \mathrm{rad} \mathrm{m}^{-2}$. The RMs could be tested with the Square Kilometer Array (SKA) and SKA pathfinders.
\end{abstract}

Keywords. large-scale structure of universe magnetic fields polarization

The nature and origin of the IGMF are not well understood. Akahori \& Ryu (2010) studied RMs through filaments of galaxies using a model of the IGMF based on MHD turbulence simulations (Ryu et al. 2008). They found that the inducement of RMs through filaments is a random walk process, and the root mean square (rms) value of the RMs for the present-day local universe is $\sim 1 \mathrm{rad} \mathrm{m}^{-2}$ (Fig. 1 left). Akahori \& Ryu (2011) studied cosmological contribution of RMs through filaments, and found that the rms value of the $\mathrm{RMs}$ reaches $\sim$ several-10 $\mathrm{rad} \mathrm{m}^{-2}$ (Fig. 1 middle). They also found that structure functions (SFs) of the RMs are $\sim 100-200 \mathrm{rad}^{2} \mathrm{~m}^{-4}$ down to $0.2^{\circ}$ scale (Fig. 1 right). Akahori et al. (2012) recently found that SFs for Galactic RMs toward high Galactic latitudes have values substantially smaller than the observed ones in angular separations less than a few degrees, suggesting that the contribution of the IGMF to the observed RMs could be significant, particularly at small angular scales. SFs at small angular scales could be tested with the SKA and SKA pathfinders with dense extragalactic sources and low instrumental noise. We suggest that image processing such as high-pass filters could be a promising method to extract the RM due to the IGMF from observed one.
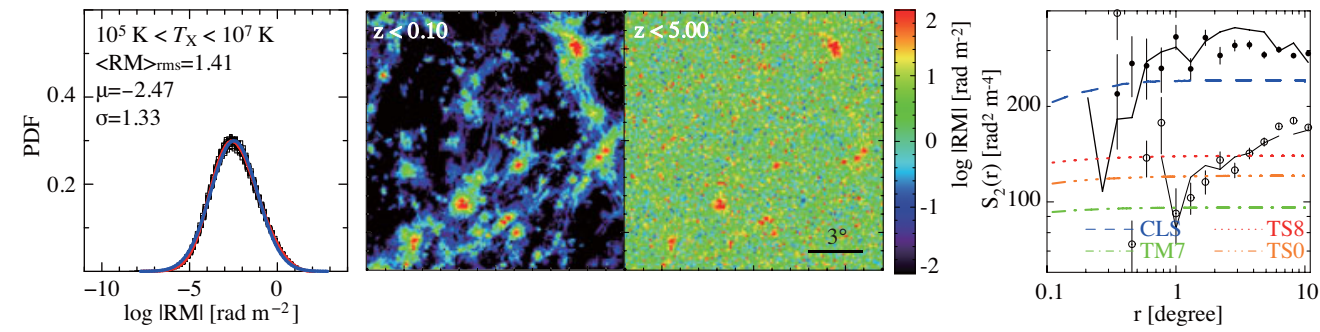

Figure 1. (Left) Probability distribution function of RMs through local filaments. (Middle) Two dimensional RM maps integrated up to redshifts of 0.1 and 5.0. (Right) SFs of RMs through filaments up to redshift of 5.0. Black lines and marks are observed SFs toward high Galactic latitudes, and color lines are expected SFs of the IGMF (see Akahori \& Ryu 2011 for details).

\section{References}

Akahori, T. \& Ryu, D. 2010, ApJ, 723, 476

Akahori, T. \& Ryu, D. 2011, ApJ, 738, 134

Akahori, T., Ryu, D., Kim, J., \& Gaensler, B. M. 2012, ApJ, submitted

Ryu, D., Kang, H., Cho, J., \& Das, S. 2008, Science, 320, 909 